\title{
Adverse Outcome in a Near Term, High-Risk Twin Pregnancy Complicated by COVID-19: A case report
}

\author{
Razmaeda Sarastry ${ }^{1 \star}$, Usissti Aladini ${ }^{1}$, Crismanto Layarta ${ }^{1}$, Alini Hafiz ${ }^{1}$, Besari Adi \\ Pramono ${ }^{1}$
}

${ }^{1}$ Department of Obstetrics and Gynecology, Diponegoro University, Semarang

Keywords:

Covid-19

Primigravida

Pregnancy

Twin Pregnancy

*) Correspondence to: razmaeda@gmail.com

Article history:

Received 30-05-2020

Accepted $22-09-2020$

Availableonline10-12-2020

\begin{abstract}
Background: COVID-19 affecting the outcome of pregnancy is still under investigation. Here we report a case of primigravida with monochorionic diamniotic twin pregnancy with superimposed preeclampsia who develop symptoms of COVID19 infection on her near term pregnancy and was confirmed later on as COVID-19 positive after cesarean section. The pregnancy was terminated abdominally due to the intrauterine fetal demise of both twins using COVID-19 standard equipment.

Case Presentation: A 29-year-old female, gravida 1, para 0 presented with a twin pregnancy at the gestational age of 36 weeks was referred to our emergency department for further management of intrauterine fetal death of both twins. Six days before admitted to our emergency department, she has been experienced a remittent fever which followed by a mild cough. Four days before admission, she complained of decrease movement of the babies. She went to the Secondary Hospital to check her pregnancy and both fetal heart rates were not found. The patient is obese (BMI $49.2 \mathrm{~kg} / \mathrm{m} 2$ ) and had prior uncontrolled hypertension but no other medical history, including epilepsy, allergies, asthma, heart disease as well as Diabetes Mellitus.

Conclusion: A research about placental pathology in pregnancy with confirmed case of COVID-19 infection showed no pathognomonic feature in the histopathologic examination but mostly, there were maternal vascular malperfusion (decidual arteriopathy in particular) and intervillous thrombi. In the end, a further examination is suggested to evaluate the course and causal factors pertaining to this case.
\end{abstract}

\section{Introduction}

COVID-19 is currently a global pandemic. Due to the high level of virus spread, pregnant women as populations at risk need special attention. However, data related to the outcome of pregnant women with COVID-19 positive are still limited.

Pregnant women with preeclampsia are included in high-risk pregnancies. The risk of preeclampsia can increase in conditions of multiple pregnancies, as well as a previous history of high blood pressure in patients. In addition, accumulating evidence reveals that hypertension is an adverse comorbid condition for COVID-19 infection.

In this report, we present a case of stillbirth babies in the twin pregnancy complicated with superimposed pregnancy who was subsequently confirmed to be COVID-19 positive.

\section{Case Presentation}

A 29 -year-old female, gravida 1 , para 0 presented with a twin pregnancy at the gestational age of 36 weeks was referred to our emergency department for further management of intrauterine fetal death of both twins. Six days before admitted to our emergency department, she has been experienced a remittent fever which followed by a mild cough. Four days before admission, she complained of decrease movement of the babies. She went to the Secondary Hospital to check her pregnancy and both fetal heart rates were not found. The patient is obese (BMI 49.2 $\mathrm{kg} / \mathrm{m} 2$ ) and had prior uncontrolled hypertension but no other 
medical history, including epilepsy, allergies, asthma, heart disease as well as Diabetes Mellitus. However, her blood pressure during routine antenatal care in the secondary Hospital was controlled using single drug alpha methyldopa and her twin were growing accordingly despite her medical history. The patient denied any impending eclampsia symptoms such as headache, epigastric pain, nausea, and blurry vision. Due to her fever history, the patient was performed thoracic X-ray which showed non-specific infiltrate on both lungs suggesting bronchopneumonia. She was then referred to our hospital for further management.
Upon arrival, her vital sign was as follow; blood pressure of 160/100 $\mathrm{mmHg}$, heart rate of 88 bpm, respiration rate 20 times per minutes $(99 \% \mathrm{O} 2$ peripheral saturation) and temperature of $36.80 \mathrm{C}$. During the clinical examination, it was found that the patient was still not in labor with cervical dilation of $2 \mathrm{~cm}$ and inadequate contractions. She was further screened for COVID-19 status using routine Early Warning Score checklist form (Table 1) and she was assessed as People at Risk (ODR: Orang Dalam Risiko) or low suspected COVID-19.

Table 1. COVID-19 Early Warning Score ${ }^{1}$

\begin{tabular}{llll}
\hline Parameters & Assessment & Finding & Score \\
\hline Signs of pneumonia on CT & Yes & not assessed & 0 \\
History of close contact with & & & \\
COVID-19 confirmed patient & Yes & No & 0 \\
Fever & Yes & No & 0 \\
Age & $>44$ years old & 29 years old & 0 \\
Sex & Male & Female & 0 \\
Tmax & $\geq 37,8^{\circ} \mathrm{C}\left(\right.$ or $\left.100^{\circ} \mathrm{F}\right)$ & No & 0 \\
Meaningful respiratory symptoms & & & \\
(including cough, expectoration, & $\geq 1$ symptom & No & 0 \\
and dyspnea & $>5.8$ & 2.92 & 0 \\
NLR & & & 0 \\
\hline Total score & & & \\
\hline
\end{tabular}

Transabdominal ultrasonography (TAUS) revealed that both fetuses were in breech presentation, both were absent of fetal movement, heart movement, and heart rate.

The fetuses presented with 2525 and 2945 grams of estimated fetal weight for first and second fetus respectively. Further, there is a bulkhead in the amniotic sacs which giving an impression of monochorionic-diamniotic twin with the single deepest pocket of amniotic fluid of $8.91 \mathrm{~cm}$. There was also found Spalding sign on both fetuses suggesting intrauterine fetal death. Placenta was implanted in the fundus, without expansion to the internal uterine ostium nor separation and rounding of placental edge.

Her laboratory data showed a white blood cell count of $10,400 / \mu \mathrm{L}$, hemoglobin level of $10,8 \mathrm{~g} / \mathrm{dL}$, hematocrit of $33,4 \%$, platelet count of $268.000 / \mu \mathrm{L}$, blood glucose of $116 \mathrm{mg} / \mathrm{dL}$, prothrombin time of $12.4 \mathrm{sec}$, activated partial thromboplastin time of 29.9 sec, aspartate aminotransferase level of $19 \mathrm{U} / \mathrm{L}$, alanine transaminase level of $10 \mathrm{U} / \mathrm{L}$, blood urea nitrogen level of $17 \mathrm{mg} / \mathrm{dl}$ and creatinine of 0.9 $\mathrm{mg} / \mathrm{dl}$, lactate dehydrogenase of $306 \mathrm{U} / \mathrm{L}$. The urine protein level was $100 \mathrm{mg} / \mathrm{dL}$.

She was administered magnesium sulfate $20 \%$ injection (1 gram/ hour on syringe pump), as well as antihypertensive agents (calcium channel blocker $10 \mathrm{mg}$ every 8 hours and alpha methyldopa $500 \mathrm{mg}$ every 8 hours). To prevent the worsening of the mother, an emergency caesarean section was performed using level 3 personal protective equipment. The infants (both female) were delivered weighing 1400 and 2700 grams for the first and second baby respectively, with an Apgar score of 0 and grade II maceration. The placenta showed no evidence of abruption such as infarct or hematoma. Her blood pressure returned to normal (110/70 $\mathrm{mmHg}$ ) right after the emergency procedure and she was admitted to the isolation room.

Day-1 following Caesarean Section, the patient's blood pressure was 130/80 $\mathrm{mmHg}$, and heart rate of $88 \mathrm{bpm}$. However, she developed another fever with a temperature of $37.60 \mathrm{C}$ which 
accompanied the complaint of dyspnea (respiration rate was 22 times per minute). Auscultation of the lungs revealed crackles on both sides but no wheezing was heard. Later, she was then consulted to The Internal Medicine Department and Microbiology Department to get the COVID-19 assessment done. She was performed nasopharyngeal swab, reevaluated for the chest X-ray, and blood was drawn for the differential count.

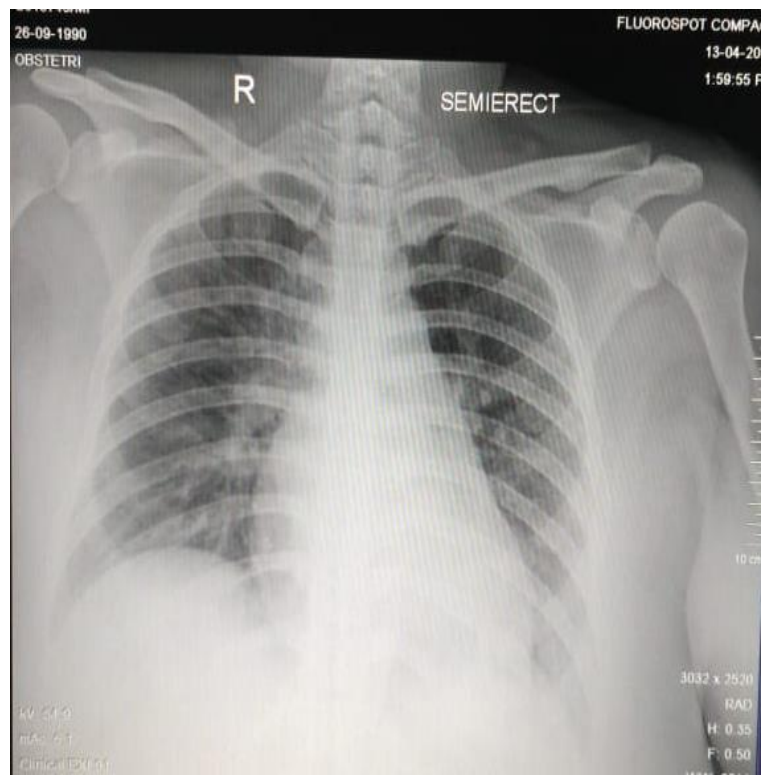

Figure 1. The chest $\mathrm{X}$-ray of the patient showed no infiltration on the lungs.

In the mean-time, she was given Oxygen via nasal cannula (3 litres per minute), antihypertensive drugs (calcium channel blocker $10 \mathrm{mg}$ every 8 hours and alpha methyldopa $500 \mathrm{mg}$ every 8 hours), vitamins (B complex, vitamin C and Sulfas Ferrous 1 tablet every 12 hours), dopamine-agonist (bromocriptine $2.5 \mathrm{mg}$ every 8 hours) and intravenous antibiotic (levofloxacin $750 \mathrm{mg}$ every 24 hours). Her subsequent chest $\mathrm{X}$-ray showed no sign of bronchopneumonia (figure 1), the differential count of white blood cells was as follows; eosinophil $0 \%$, basophil $0 \%$, segmented-neutrophil 73\%, lymphocyte $25 \%$, and monocyte $1 \%$ (NLR of 2.92).

Day-3 following the Cesarean section, the patient's vital sign was improved with the blood pressure of 130/80 $\mathrm{mmHg}$, heart rate $98 \mathrm{bpm}$, the respiration rate of 20 times per minute, and temperature of $37 \mathrm{oC}$. She was discharged on the $3 \mathrm{rd}$ day post-operation according to clinical practice in the hospital. One week after discharge, her swab test was positive for COVID-19. She was then admitted to the District Hospital for isolation and treatment with current stable conditions.

\section{Discussion}

SARS-CoV2 virus or novel Coronavirus is single-stranded RNA, non-segmented, enveloped viruses, which cause a wide range of illnesses from the common cold to severe disease. Since the emergence of this virus, there is little data on adverse pregnancy outcomes in pregnant women with COVID-192,3. Previous studies on other coronaviruses (SARS-CoV and MERS-CoV) proposed that pregnancy poses a higher risk of severe illness, morbidity, and mortality compared with the general population2. However, to our knowledge, there has been no case report that describes the stillbirth of near-term, twin pregnancy complicated with symptomatic COVID-19 infection.

Viral pneumonia has been described as an important cause of morbidity and mortality during pregnancy. It is associated with adverse obstetrical outcomes, such as premature rupture of membranes (PROM) and preterm labor (PTL), intrauterine fetal demise (IUFD), intrauterine growth restriction (IUGR) as well as neonatal death 3 . However, it is not evident that these outcomes were related to maternal infection, and the risk of adverse infant outcomes is still obscure2,3. Currently, very limited is known about COVID-19 related to its effect on pregnancy and neonatal period. Furthermore, there are no specific recommendations for pregnant women regarding the evaluation or management of COVID-19.

It has been suggested that twin gestations have a higher risk for prospective IUFD4. Furthermore, monochorionic twin gestations have higher rates of stillbirth than either dichorionic twin or singleton gestations5. This is attributed primarily to placental vascular complications such as twin to twin transfusion syndrome. Other factors include selective growth restriction, maternal obstetric complications as well as co-twin death due to intravascular shunting after a single intrauterine death6. A study involved a total of 454,626 twins showed that stillbirth was observed in 1585 fetuses $(0.35 \%)$ overall. However, the risk of fetal demise was statistically significant only between 37 and 38 weeks' gestation 4 . Additionally, a study on 768 twins showed the prospective risk of IUFD at $\geq 34$ weeks' gestation was $0 \%$ and $0.17 \%$ for monochorionic and dichorionic twins respectively5.

Regarding hypertensive disorders during pregnancy, the risk of stillbirth in singleton pregnancy was significantly higher than in normotensive women7,8. In contrast, in multifetal pregnancy, stillbirth was generally not significantly associated with hypertensive disorders. With only 
superimposed preeclampsia was associated with a significantly increased risk of stillbirth by 1.95 fold overall8. Furthermore, this increase was observed only at $\leq 28$ weeks' gestation and at 28 to 31 weeks' gestation8.

Owing to the findings of our patient who developed superimposed preeclampsia at a near term pregnancy (36 weeks), the possibility that twin fetal demise was due to complications of COVID-19 is still open on the table. However, there is no histopathologic examination of the placental tissue that might be responsible to be the cause of the complication to this pregnancy. A research about placental pathology in pregnancy with confirmed case of COVID-19 infection showed no pathognomonic feature in the histopathologic examination but mostly, there were maternal vascular malperfusion (decidual arteriopathy in particular) and intervillous thrombi. ${ }^{9}$ In the end, a further examination is suggested to evaluate the course and causal factors pertaining to this case.

\section{Limitation}

This study had limitation due to there were no further examination of the demised babies such as pathological and RT PCR to rule out the vertical transmission of COVID-19.

\section{References}

1. Song C-Y, Xu J, He J-Q, Lu Y-Q. COVID-19 early warning score: a multi-parameter screening tool to identify highly suspected patients. 2020;
2. Infecting CS-, Graham AL. Potential Maternal and Infant Outcomes from Coronavirus 2019nCoV (SARS-CoV-2) Infecting Pregnant Women: Lessons from SARS, MERS, and Other Human Coronavirus Infections. 2020;1-16.

3. Karimi-zarchi M, Neamatzadeh H, Dastgheib SA, Abbasi H, Mirjalili SR, Behforouz A, et al. Vertical Transmission of Coronavirus Disease 19 (COVID-19) from Infected Pregnant Mothers to Neonates: A Review Vertical Transmission of Coronavirus Disease 19 (COVID-19) from Infected Pregnant Mothers to Neonates: A Review. Fetal Pediatr Pathol. 2020;0(0):1-5.

4. Page JM, Pilliod RA, Snowden JM, Caughey $\mathrm{AB}$. additional week of expectant management in twin pregnancies. Am J Obstet Gynecol. 2015;212(5):630.e1-630.e7.

5. Burgess JL, Unal ER, Nietert PJ, Newman RB. Risk of late-preterm stillbirth and neonatal morbidity for monochorionic and dichorionic twins. Am J Obstet Gynecol. 2014;210(6):578.e1-578.e9.

6. Ortiz JU, Martinez JM. A Systematic Approach to the Differential Diagnosis and Management of the Complications of Monochorionic Twin Pregnancies. 2012;145-55.

7. Harmon QE, Huang L, Umbach DM, Engel SM, Magnus P, Zhang J, et al. HHS Public Access. 2016;125(3):628-35.

8. Xiong T, Mu Y, Liang J, Zhu J, Li X, Li J, et al. Hypertensive disorders in pregnancy and stillbirth rates: a facility- based study in China. 2018;(May):531-9.

9. Shanes ED, Mithal LB, Otero S, Azad HA, Miller ES, Goldstein JA. OUP accepted manuscript. Am J Clin Pathol. 2020;1-10. 